\title{
Group-ICA with Functional Connectivity During Inhibition Control in Young Adults With Autistic-like Traits: an fMRI Study of a Stop-Signal Task
}

Yaxu Yu

Southwest University

$\mathrm{Li} \mathrm{He}$

Southwest University

qiu jiang ( $\square$ qiuj318@swu.edu.cn )

Southwest University https://orcid.org/0000-0003-0269-5910

\section{Research}

Keywords: autistic-like traits, response inhibition control, stop-signal task, groupICA

Posted Date: November 23rd, 2020

DOI: https://doi.org/10.21203/rs.3.rs-111822/v1

License: (c) (i) This work is licensed under a Creative Commons Attribution 4.0 International License.

Read Full License 


\section{Abstract}

\section{Background}

Few studies explored response inhibition in autistic-like traits people, using task fMRI. In this study, we examine the functional connectivity of the brain using a stop-signal task based on fMRI among young adults with autistic-like traits and investigated their ability to achieve inhibition control.

\section{Methods}

29 of Chinese individuals measured with AQ. Then applied stop signal task to explore the difference in brain functional connectivity in individuals with autistic-like traits.

Results

The results showed autistic-like traits people the longer the SSRT, the worse the inhibition ability. And we used networks obtained from groupICA analysis at the functional connectivity analysis level, the SN had a negative connection with left SMG; the DAN had a negative connection with left LG; the FPN had a positive connection with left PCG; the LN had a positive connection with vermis 45 and negative connection with left ITG. Furthermore, the SMG, LG, PCG, and temporal gyrus were also obtained in ROIto-ROI analysis.

\section{Limitations}

Our sample size smaller, still need to multicenter, large sample confirmed this conclusion. We want to use more task paradigms to explore inhibition control in autistic-like traits people.

\section{Conclusions}

We found that autistic-like traits people had atypical functional connectivity within brain networks in the SN, DAN, FPN, and LN, and had atypical brain areas centered on the SMG, LG, PCG, and temporal gyrus. And also highlight the importance of considering executive control function of whole-brain functional connections to better characterize brain connectivity in young adults with autistic-like traits.

\section{Background}

"Autistic-like traits" is a term describing a group of subliminal social skills and communication traits and unusual personality features that are believed to be milder manifestations of traits characteristic for clinically diagnosed autism (Constantino et al., 2006; Rutter, 2000). The continuity of population distribution clearly identified the character of autism spectrum disorders (ASD), since the DSM-5 no longer classified ASD in 2013 (A. P. Association, 2013). In other words, the classification of autistic-like traits changed from a categorical classification with or without autism to a quantitative classification with continuity, which also reflects the diversity and heterogeneity to some extent (Lai, Lombardo, 
Chakrabarti, \& Baron-Cohen, 2013). This group is also thought to have a disability in inhibition control (Agam, Joseph, Barton, \& Manoach, 2010; R. K. Kana, T. A. Keller, N. J. Minshew, \& M. A. Just, 2007; Rinehart, Bradshaw, Moss, Brereton, \& Tonge, 2008). This disability may adversely impact their daily life, mental health and productivity (Foss-Feig et al., 2017). With the development of neuroscience and anatomy, numerous studies showed the inhibition process involved in some brain areas, such as the corpus callosum (Alexander et al.; Giuliano et al., 2018; Just, Cherkassky, Keller, Kana, \& Minshew, 2006), thalamus (Daly et al., 2014; Haznedar et al., 2006), anterior cingulate cortex (Chmielewski, Yildiz, \& Beste, 2014), prefrontal cortex (T. Xiao et al., 2012), temporal gyrus (Sheikhani, Behnam, Mohammadi, Noroozian, \& Mohammadi, 2012), and parietal gyrus (Vara et al., 2014). In addition to abnormalities in voxel-based brain areas, functional connectivity (FC) was also found in specific brain networks. In Just's study, ASDs in inhibition control processes have underconnectivity within brain networks, mostly focused on the functional connectivity between frontal gyrus and other brain areas, especially the FC between frontal-parietal and frontal to posterior brain regions (Di Martino et al., 2009; Di Martino et al., 2013; McKinnon et al., 2019; Shih et al., 2011). Kana used Functional magnetic Resonance Imaging (fMRI) investigate inhibition control ability in ASDs using a go/no-go task, and the results showed poorer integration between the anterior cingulate cortex and frontal-parietal network (FPN), and decreased FC between them (Rajesh K. Kana, Timothy A. Keller, Nancy J. Minshew, \& Marcel Adam Just, 2007).

Some studies involved in response inhibition indicated that patients with ASDs are impaired in terms of inhibiting responses (Adams \& Jarrold, 2009; Bishop \& Norbury, 2005; Lemon, Gargaro, Enticott, \& Rinehart, 2011; Semrud-Clikeman, Walkowiak, Wilkinson, \& Butcher, 2010; Vaidya et al., 2011; T. Xiao et al., 2012). Other studies claimed that patients with ASDs have deficits in repetitive behavior that might be caused by nondevelopment in cognitive control functions (Langen, Leemans, Johnston, Ecker, Daly, Murphy, dell'Acqua, et al., 2012; Mosconi et al., 2009). Most studies focused on ASD applied many classic experiment paradigms. The stop-signal task (SST) is one of the most widely used (Geurts, van den Bergh, \& Ruzzano, 2014). Many studies involved in children (Schmitt, Ankeny, Sweeney, \& Mosconi, 2016), young adults (Ting Xiao et al., 2012), and adults (Wilson et al.) with ASD, all with impairment in cognitive control ability. In an ASD study, the ASD group and control group both showed decreased dorsal anterior cingulate cortex activation (dACC) consistent with a role in top-down control, associated with a higher error rate. The ASD group showed that both brain activation and functional connectivity were associated with more severe restricted repetitive behavior (Agam et al., 2010). The other study involved neurocognitive mechanisms of the response delay effect, and the results showed that active braking might explain the neurological mechanisms of the response delay effect at least, which may be similar to the mechanism of stopping responses completely. These results further explain how individuals point to proactive recruitment of a neurocognitive mechanism when responding with restraint, associated with outright stopping (Jahfari, Stinear, Claffey, Verbruggen, \& Aron, 2010). Another study used color-word interference tests, trail making tests, verbal fluency tests, and design fluency tests to explore flexibility, fluency, and inhibition in autism and Asperger's disorder. The results showed that participants performed significantly below average on a composite measure of executive functioning adjusted for baseline 
cognitive ability (Kleinhans, Akshoomoff, \& Delis, 2005). Schmitt used the stop-signal task and showed that ASD had lower correct rates and more reaction time in stop trials (Schmitt et al., 2016).

In addition to involvement in nontraditional development of neurological mechanisms and poor behavior performance, there are also age-related characteristics (Padmanabhan et al., 2015). Typical development (TD) people have cognitive control ability that improve throughout childhood, and reaches adult levels at age 15, they tend to delay behavior actively, rather than inhibiting it passively when in the young adult state (Luna, Doll, Hegedus, Minshew, \& Sweeney, 2007; Vink et al., 2014). ASD have similar development and maturation as TD people, while patients with ASD have obvious deficits in cognitive control ability when in the adolescent and adult state (Geurts et al., 2014). Young adults are thought to be in a significant period of development of cognitive control ability (Humphrey \& Dumontheil, 2016; Oberle, Schonert-Reichl, Lawlor, \& Thomson, 2012). Padmanabhan's study used an anti-saccade task and visually guided saccade task combined with $\mathrm{fMRI}$ to investigate the difference of inhibition control ability between young adults and adults with high functioning autism TD. The results showed that cognitive control ability did not improve with age in the ASD group. An fMRI analysis revealed that in young adults with ASD, the frontal eye field was not activated in the anti-saccade task, in which key brain areas of the inhibition control should be needed, while the putamen was found to have greater activation. This result suggested that the frontal eye field has delayed development in ASD compared with that of TD (Padmanabhan et al., 2015).

As mentioned above, the young adult state is critical in the development of cognitive control. Young adults with ASD might have different activation of brain circuits and functional connectivity, with latent development deficits in cognitive control. During the young adult state, which is a key period of brain maturation, brain functions in ASD might not achieve the key transformation. Most studies focused on ASD patients, while rarely have studies explored inhibition control ability in individuals with autistic-like traits, especially in young adults. In the present study, we explored young adults in normal population with autistic-like traits using a stop-signal task of fMRI, to apply a data-driven method (group-ICA analysis) to identify independent network components, in inhibitory conditions. Then, based on group-ICA results, we took group-ICA network (independent components IC) as a seed, and applied network as a seed to whole-brain connectivity analysis, in order to explore more detailed patterns of autistic-like traits linked functional connectivity in the inhibitory state. Next, a region of ROI-to-ROI analysis was used to explore the neurological mechanisms of brain areas and functional connectivity. Pearson analysis was performed to investigate the relationships between subdimensions of autism spectrum quotient (AQ) and the values of brain areas.

\section{Methods}

\section{Participants}

A total of 39 people were recruited for this study. 30 subjects with fMRI task results matched to the AQ participated in this study. Due to excessive head movements, 1 participant was excluded (> $3 \mathrm{~mm}$ 
translation and angular rotation in each axis). Thus, a total of 29 (men: 11; mean age: 19.21 y, SD: 0.41, range: 19-20 y) individuals participated in this research. All methods were in accordance with the Declaration of Helsinki (W. M. Association, 1991). All participants were right-handed, with no history of neurological or psychiatric problems. All participants provided written informed consent and received payment for their time. The study was approved by the Southwest University Brain Imaging Center Institutional Review Board.

\section{Assessment of the AQ}

The AQ was used to measure autistic-like traits in individuals from the general population (Baron-Cohen, Wheelwright, Skinner, Martin, \& Clubley, 2001). The Chinese version of the AQ (Lau et al., 2013) was used in this study, which consists of tSocial Skill, Communication, Attention Switching, Imagination and Attention to Detail subscales, as represented by fifty statements; participants responded to these statements on a 4-point Likert scale, with "definitely agree" and "slightly agree" responses being scored one point and "slightly disagree" and "definitely disagree" responses being scored one point among the reverse-scored options. In half of the statements, the diagnostic answer was "agree," and in the other half, it was"disagree." One point is awarded for each diagnostic answer, which resulted in a continuous distribution of scores in the population sample. The total score ranged from 0 to 50 points, with higher scores suggesting a higher magnitude of autistic-like traits. Currently available data from research on the properties of this scale indicate that the measurement reliability for the total score is satisfactory (Austin, 2005; Hoekstra, Bartels, Cath, \& Boomsma, 2008; Hurst, Mitchell, Kimbrel, Kwapil, \& Nelson-Gray, 2007; Ingersoll, Hopwood, Wainer, \& Brent Donnellan, 2011; Kloosterman, Keefer, Kelley, Summerfeldt, \& Parker, 2011). In the present study we focused on analyzing the total $A Q$ score.

\section{Stop-Signal Task}

The stop signal task (Aron \& Poldrack, 2006) consists of 32 Stop trials and 96 Go trials. Each trial began with a central fixation cross over $300 \mathrm{~ms}$, and a green arrow pointing left or right was subsequently displayed on a screen. For the Go task, the green arrow lasted for $500 \mathrm{~ms}$, and participants were asked to respond as fast and accurately as possible by pressing the " 1 " when appeared green left arrow, or " 2 " button when appeared green right arrow within $1500 \mathrm{~ms}$, then the blank screen lasted for $700 \mathrm{~ms}$. For the Stop task, the stop signal delay (SSD) as the duration of the green arrow, which was dynamically adjusted according to the participant's response, then the stop signal (red arrow) appeared for $300 \mathrm{~ms}$, and participants were asked to withhold the response they already initiated. Specifically, the initial SSD value was $250 \mathrm{~ms}$. If the participant inhibited successfully on a Stop trial, then inhibition was made more difficult on a subsequent stop trial by increasing the SSD by $50 \mathrm{~ms}$; if the participant did not successfully inhibit, then inhibition was made easier by decreasing the SSD by $50 \mathrm{~ms}$. Next, we subtracted the SSD from $1200 \mathrm{~ms}$ as the duration of the blank screen. The jitter between trials ranged from $1 \mathrm{~s}$ to $4 \mathrm{~s}$ (mean, 
$2.5 \mathrm{~s})$. The duration of the task was $8 \mathrm{~min}, 46 \mathrm{~s}$. The instructions, the presentation, and a fixation cross were included in each trial (see Figure 1).

\section{MRI Data Acquisition and Preprocessing}

All subjects underwent MRI scanning at the Brain Imaging Center of Southwest University. Whole-brain imaging data were obtained using a 3.0-T Siemens Trio MRI scanner with a 12-channel whole-brain coil, functional images including 32 continuous slices were obtained with a T2*-weighted (Siemens Medical, Erlangen, Germany). For each subject, 263 blood oxygen level-dependent (BOLD) images were acquired during the $\mathrm{fMRI}$ task with a gradient echo type echo planar imaging (EPI) sequence [echo time $(T E)=30$ $\mathrm{ms}$; repetition time $(T R)=2000 \mathrm{~ms}$; flip angle $=90$ degrees; slice thickness $=3.0 \mathrm{~mm}$; slices $=32$; resolution matrix $=64 * 64$; voxel size $=3.4 * 3.4 * 4 \mathrm{~mm}$; field of view $(F O V)=220 \times 220 \mathrm{~mm}^{2}$; thickness $/$ inter slice gap $=3 / 1 \mathrm{~mm}$; and sampling bandwidth $=250 \mathrm{kHz}$.

Preprocessing was performed using the Data Processing Assistant for Resting-state fMRI (DPARSF; http://rfmri.org/DPARSF) (Yan \& Zang, 2010), which is a toolbox based on the SPM8 software package (www.fil.ion.ucl.ac/spm). The functional data were realigned and unwrapped to correct for head movement artifacts based on field maps. Whole images underwent temporal processing and slice timing and motion correction to reduce displacement between volumes, spatial normalization to the standard Montreal Neurological Institute (MNI) space with a resampled voxel size of $3 \times 3 \times 3 \mathrm{~mm}^{3}$ using the T1 scans as reference images, and spatial smoothing ( $4 \mathrm{~mm}$ full width half maximum, FWHM), band-pass temporal filtering $(0.01-0.1 \mathrm{~Hz})$. To minimize the effects of head motion, subjects were excluded maximal motion between volumes in each direction $>3 \mathrm{~mm}$, and rotation about each axis $>3^{\circ}$.

\section{Group Independent Component Analysis and Component Selection}

Group independent component analysis (ICA) was performed in the preprocessed fMRI data using CONN 18b (Whitfield-Gabrieli \& Nieto-Castanon, 2012) with G1 FastICA and GICA3 back-projection in Calhoun's group-ICA algorithm (Calhoun, Adali, Pearlson, \& Pekar, 2001), which based on all subjects and all conditions. The number of components we used was 25 through MDL analysis to identify independent components (IC) of cortical and subcortical brain areas that corresponds to functional segmented networks. The timecourses and spatial maps were normalzied into z-scores (Beckmann, DeLuca, Devlin, \& Smith, 2005).

16 other ICs were discarded as eyemovements, head motion, cardiac-induced pulsatile artifacts, psychological noise, movement signals, or artifacts of canner (Cordes et al., 2000). 9 ICs inclueded 6 subnetworks identified by the CONN network cortical ROIs (HCP-ICA) through the ICA analysis. These subnetworks were the default mode network (DMN), salience network (SN), dorsalattention network (DAN), frontoparietal network (FPN), language network (LN), and cerebellar network (CN). 


\section{Take Network as A Seed to Whole-brain Connectivity Analysis}

In the first-level analysis, general linear model (GLM) analysis was used to separately estimate the effects in the three conditions (go task, stop task and base), implemented by boxcar functions convolved with a canonical hemodynamic response function (HRF) performed using the CONN 18b toolbox (Cognitive and Affective Neuroscience Laboratory, Massachusetts Institute of Technology, Cambridge, MA, USA; www.nitrc.org/projects/conn). In addition, age, gender and six head motion parameters were taken as confounders. Then, we calculated "contrast images" for each participant for the stop success and base conditions and then computed the whole-brain activity pattern for this contrast, through which subjectspecific effects were estimated using the linear contrasts obtained from the first-level analysis. All fMRI data analyses were performed using SPM8 (www.fil.ion.ucl.ac/spm).

To identify specific brain regions that are more positively or negatively connected to the seed network in inhibitory condition among young adults with autistic-like traits, the seed network to whole-brain connectivity was performed using the CONN toolbox. The seed networks used were based on spatial properties in ICA analysis. The onsets and durations of the task were delineated in order to measure connectivity between the networks and the rest of the brain during each condition of the scanning period. The beta maps were created for each individual at whole session. Only the beta maps that measured network connectivity during stop-signal task "stop success $>$ base" period were entered into second-level analysis in CONN. The spatial masks of cerebrospinal fluid (CSF), white matter, ART-based scrubbing, and 6 rigid-body parameters were regressed out of whole-brain gray matter activity. To reduce the effect of low-frequency drift and high-frequency noise, bandpass filtering $(0.01<f<0.1 \mathrm{~Hz})$ was used. The resulting maps were thresholded at a $p<0.05$ ( $p$-uncorrected voxel threshold) and $p<0.05$ ( $p$-FDR (false discovery rate) corrected cluster-size).

\section{ROI-to-ROI Analysis}

To explore more detailed patterns of autistic-like trait-linked functional connectivity in the inhibitory state, all pairwise connections between Power 264 cortical brain regions (Power et al., 2011) and 30 suncortical brain regions from Human Brainnetome Atlas (Fan et al., 2016) were exainmed for subsets of brain regions that have significant connection to one another's seeds. This approch use ROI-to-ROI analysis in CONN toolbox at second level, age and gender as covariates, A FDR-corrected $p<0.05$ at the seed-level was adopted in this study.

\section{Correlation Analysis Between AQ Total Score and Subdimentions and brain areas}


To identify those dimensions of $A Q$ that have specific close relationships with brain areas, we applied correlation analysis between $\mathrm{AQ}$ and the brain values extracted taken from the network as seed-to-voxel of functional connectivity analysis results.

\section{Results}

\section{Behavioral Results}

The demographic data and behavioral results are shown in Table. 1. The mean AQ score of the current sample was 21.83 , and the standard deviation was 4.86 .

\section{Stop-Signal Task Performance}

In this study, we used stop signal reaction time (SSRT) as an important role to measure people's ability in terms of response inhibition: the longer the SSRT, the worse the inhibition ability (Logan, Cowan, \& Davis, 1984). The results showed a significant positive association between AQ and SSRT, which means that young adults with high levels of autistic-like traits, have relatively weaker inhibition control, $(p<0.05$; Table 2).

\section{Take Network As a Seed to Whole-brain Connectivity Results}

Six networks default mode network (DMN), salience network (SN), dorsal attention network (DAN), frontoparietal network (FPN), language network (LN), and cerebellar network (CN) from group-ICA analysis were used as seeds in a whole-brain search to identify specific brain regions that associated with young adults with autistic-like traits in stop success vs base conditions. The results showed that four networks contained SN, DAN, FPN, and LN have significant functional connectivity with other brain areas. Specifically, the SN (IC13, independent component13) had a significant negative connection with left supramarginal gyrus (SMG); the DAN (IC20) had a significant negative connection with left lingural gyrus (LG); the FPN had a significant positive connection with left postcentral gyrus (PCG); the LN (IC7) had a significant positive connection with vermis 45 and negative connection with left inferior temporal gyrus (ITG), (Table 3 and Figure 2).

\section{ROI-to-ROI Results}

We obtained more detailed brain areas of autistic-like traits linked functional connectivity in the inhibitory state. At the ROI-to-ROI level, we found three negative connections and four positive connections, including SMG, postcentral gyrus (PCG), lingual gyrus (LG), and temporal gyrus. These four brain areas 


\section{Correlation Results Between AQ and Subdimentions and Brain areas}

The correlation results showed that the social skill of AQ had a significant positive relationship with PCG; attention switching of $A Q$ had a significant positive relationship with PCG, and significant negative relationship with ITG and SMG; attention to detail had a significant negative relationship with ITG; communication had a significant positive relationship with PCG, and significant negative relationship with ITG, SMG and LG (Table 5).

\section{Discussion}

In this study, we examined the atypical functional connectivity in young adults with autistic-like traits when processed response inhibition using stop-signal tasks. The results showed that as the AQ score increased, young adults showed longer the SSRT, which means worse response inhibition control ability. In primary analysis, used a groupICA approach and obtained six brain networks, DMN, SN, DAN, FPN, LN, and $\mathrm{CN}$. And then taken these networks as seeds to the whole brain approach and found some established connectivity networks showing greater significant connections to the left SMG, left LG, left PCG, verimis4 5 and left ITG. ROI-to-ROI analysis showed multiple significant connections, while the left SMG, left LG, left PCG and temporal gyrus were also included in this results, and were indeed important for autistic-like traits associated connectivity.

\section{Stop-Signal Task Performance}

The results of the stop signal task performance of the young adults with autistic-like traits showed that the higher AQ score was, the longer the response time of SSRT was. The increase of response time meant poor inhibition and control ability of people with autistic-like traits. Quantitative studies showed that inhibition control ability is one of the core components of executive function (Chung, Weyandt, \& Swentosky, 2014; Geurts et al., 2014; Langen, Leemans, Johnston, Ecker, Daly, Murphy, dell'Acqua, et al., 2012). Individuals with better inhibitory control ability avoid some improper or adverse reactions in processing cognitive control task (Coxon, Stinear, \& Byblow, 2007). In the process of complex social communication, the ability to screen out unimportant information and focus on critical information has pivotal effects from childhood to adulthood, and these effects are continuous (Barendse et al., 2013; Carlson \& Moses, 2001; Carlson, Moses, \& Breton, 2002; Hala, Hug, \& Henderson, 2003; Pelphrey, Morris, \& McCarthy, 2004). 


\section{Atypical Functional Connectivity in Young Adults with Autistic-like Traits in Response Inhibition Control Ability}

Taking network as seed-to-voxel analysis showed that LN, DAN, FPN, and LN had significant connections with whole-brain areas. First, LN had increased functional connectivity with vermis4 5, and decreased functional connectivity with ITG. Many studies showed that the vermis always had a close relationship with ASD, from brain structure (Hashimoto, Tayama, Miyazaki, Murakawa, \& Kuroda, 1993) to functional connectivity (Wang et al., 2019). A study involving ASD showed that the vermis had significant negative association with eye fixation time, suggesting that the vermis plays a key role in processing visual information (Laidi et al., 2017). The results also found that the LN had a decrease connection with ITG. The ITG plays an important role in LN, and decreased functional connectivity suggested that young adults with autistic-like traits have semantic function that is different from that of traditional people (Herringshaw, Ammons, DeRamus, \& Kana, 2016).

We also found SN had decreased functional connectivity with SMG. The SMG is a part of the superior parietal lobule (SPL) (Stoeckel, Gough, Watkins, \& Devlin, 2009). The SN is responsible for screening received information and then allocating it to other functions of the brain. The SPL is part of the SN (Bressler \& Menon, 2010; Seeley et al., 2007). Decreased within-network functional connectivity in the SN suggests that it does not play a fully role in allocating stimuli (Winston et al., 2013).

In addition, the DAN had decreased functional connections with the LG, providing top-down attention orientation (Lei, Wang, Yuan, \& Mantini, 2014). . In task states, individuals were prompted to respond in what form, where and when; the DAN was continuously activated to ensure the task was completed well (Bogousslavsky, Miklossy, Deruaz, Assal, \& Regli, 1987). The LG is responsible for visual memory, visual representation and involved in formation of visual image (Belardinelli et al., 2009). Decreased functional connectivity between the DAN and the LG suggested that young adults with autistic-like traits did not concentrate well during the process of response inhibition task, leading to longer SSRT in young adults with autistic-like traits.

Finally, the right FPN has increased functional connectivity with the PCG. The right FPN is responsible for executive control (Duan et al., 2017; Just, Cherkassky, Keller, Kana, \& Minshew, 2007; Smallwood, Brown, Baird, \& Schooler, 2012), while the PCG is one the main brain areas of the sensorimotor system (Corkin, Milner, \& Rasmussen, 1970; Paakki et al., 2010), and this connection suggests that young adults with autistic-like traits when processing response inhibition task might be disturbed by other functional regions, and they might not avoid this interference effectively. We also found these four brain areas appeared in ROI-ROI analysis, which also suggests that these four brain areas play specific pivotal roles and might also be hallmarks of executive control in young adults with autistic-like traits.

\section{Subdimensions of AQ Have an Association with Specific Brain Areas}


The correlation results showed that, first, social skill, attention switching, and communication of $A Q$ had a significant positive relationship with the PCG. The PCG is responsible for sensorimotor brain function (Corkin et al., 1970; Paakki et al., 2010), and this suggests that young adults with autistic-like traits, whose typical characteristics include stereotyped behavior, might not screen out irrelevant stimuli well in the inhibition task state. This finding means that increased functional connectivity with the PCG can predict the severity of social skill deficits at some point. We then found that attention switching, attention to detail, and communication had significant negative relationships with the ITG. The ITG is included in the language network and has a key role as a bridge in performing various tasks (Herringshaw et al., 2016). The LN is constantly adjusted in task processing, the previously activated parts are suppressed, and the newly activated parts keep appearing until the task is successfully completed (Sachs et al., 2008). The decreased functional connectivity with the ITG also suggests that young adults with autisticlike traits have deficits in linkage mechanisms of the brain networks, possibly reducing the degree of cooperation between networks. The third one was that attention switching and communication of $A Q$ had decreased functional connectivity with the SMG, and the last one was communication of $A Q$ had decreased functional connectivity with the LG. The SMG is included in the superior parietal lobule (SPL) (Stoeckel et al., 2009), which is involved in executive control and attention shifting (de Wit, 2018; Takarae, Luna, Minshew, \& Sweeney, 2014). The decreased tendency in the SPL indicated that the deficits of these areas may explain the nonsocial difficulties in individuals with autistic-like traits, such as repetitive, poorly controlled, poor goal-directed action (Salmi et al., 2013). The LG is responsible for visual memory and visual representation and is involved in the formation of visual image (Belardinelli et al., 2009). The decreased functional connection with the LG suggests that young adults with autistic-like traits have poor inhibition control ability and that they might not concentrate well during the process of response inhibition tasks

\section{Limitations}

In the present study, we investigated response inhibition control ability using stop-signal task of $\mathrm{fMRI}$ in individuals with autistic-like traits. The results showed that individuals with autistic-like traits have deficits in response inhibition control ability and have atypical functional connectivity. While our sample size smaller, still need to multicenter, large sample confirmed this conclusion. We want to use more classical experimental paradigms like go\no go task, stroop task to explore autistic-like traits people'different types of executive functions in the future study.

\section{Conclusion}

The current findings support adopting a mainstream perspective to help reconcile the heterogeneous findings of functional connectivity in young adults with autistic-like traits. These results demonstrated differences specific to certain ages, highlighting the utility of carefully considering age in studies of functional connectivity in autistic-like trait groups. We found that young adults with autistic-like traits had atypical functional connectivity within brain networks in the SN, DAN, FPN, and LN, and had atypical 
brain areas centered on the SMG, LG, PCG, and temporal gyrus. Post-hoc analysis found that subdimensions of $A Q$ had significant associations with functional connectivity of brain areas. Both brain networks and brain regions in young adults may help to explain some of the altered functions often found in young adults with autistic-like traits. These results also highlight the importance of considering executive control function of whole-brain functional connections to better characterize brain connectivity in young adults with autistic-like traits.

\section{Abbreviations}

Group-ICA: Group Independent Component Analysis

IC: independent components

fMRI: Functional magnetic Resonance Imaging

AQ: Autism spectrum Quotient

SSRT: stop signal reaction time

FC: functional connectivity

DMN: networks default mode network

SN: salience network

DAN: dorsal attention network

FPN: frontoparietal network

LN: language network

$\mathrm{CN}$ : cerebellar network

SMG: supramarginal gyrus

LG: lingural gyrus

PCG: postcentral gyrus

ITG: inferior temporal gyrus

ROI: Region of Interests

GLM: general linear model

HRF: hemodynamic response function 


\section{Declarations}

\section{Ethics approval and consent to participate}

All methods were in accordance with the Declaration of Helsinki. All participants provided written informed consent and received payment for their time. The study was approved by the Southwest University Brain Imaging Center Institutional Review Board.

\section{Consent for publication}

No application

\section{Availability of data and materials}

The datasets used and analysed during the current study are available from the corresponding author on reasonable request

\section{Competing interests}

The authors declared no competing interests.

\section{Funding}

This research was supported by the National Natural Science Foundation of China (31771231, 32071070), Natural Science Foundation of Chongqing (cstc2019jcyj-msxmX0520, cstc2020jcyjmsxmX0299), the planned project of Chongqing humanities and Social Sciences (2018PY80, 2019PY51), and Fundamental Research Funds for the Central Universities (SWU119007), Chang Jiang Scholars Program, National Outstanding Young People Plan, Chongqing Talent Program.

\section{Author contributions}

Yu and Wang conducted the experiments, analyzed the results and carried on paper writing, Qiu proposed many constructive advises in manuscript. All authors reviewed the manuscript.

Yaxu Yu and Li He contributed equally to this work. Qiu is the corresponding author.

\section{Acknowledgements}

We thank all the participants who take part in this study. We also thank our Professor Qiu provide ideas, modification and spiritual help.

\section{References}

Adams, N. C., \& Jarrold, C. (2009). Inhibition and the validity of the Stroop task for children with autism. $J$ Autism Dev Disord, 39(8), 1112-1121. doi:10.1007/s10803-009-0721-8 
Agam, Y., Joseph, R. M., Barton, J. J., \& Manoach, D. S. (2010). Reduced cognitive control of response inhibition by the anterior cingulate cortex in autism spectrum disorders. Neuroimage, 52(1), 336-347. doi:10.1016/j.neuroimage.2010.04.010

Alexander, A. L., Lee, J. E., Lazar, M., Boudos, R., DuBray, M. B., Oakes, T. R., . . McMahon, W. M. Diffusion tensor imaging of the corpus callosum in Autism. Neuroimage, 34(1), 61-73.

Aron, A. R., \& Poldrack, R. A. (2006). Cortical and subcortical contributions to stop signal response inhibition: role of the subthalamic nucleus. Journal of Neuroscience, 26(9), 2424-2433.

Association, A. P. (2013). Diagnostic and statistical manual of mental disorders (DSM-5®): American Psychiatric Pub.

Association, W. M. (1991). Declaration of Helsinki. Law, medicine \& health care: a publication of the Amercian Society of Law \& Medicine. Gastroenterologia Japonica, 26(2), 269-270.

Austin, E. J. (2005). Personality correlates of the broader autism phenotype as assessed by the Autism Spectrum Quotient (AQ). Personality and Individual Differences, 38(2), 451-460.

Barendse, E. M., Hendriks, M. P., Jansen, J. F., Backes, W. H., Hofman, P. A., Thoonen, G., . . Aldenkamp, A. P. (2013). Working memory deficits in high-functioning adolescents with autism spectrum disorders: neuropsychological and neuroimaging correlates. Journal of neurodevelopmental disorders, 5(1), 14.

Baron-Cohen, S., Wheelwright, S., Skinner, R., Martin, J., \& Clubley, a. E. (2001). The Autism-Spectrum Quotient (AQ): Evidence from Asperger Syndrome/High-Functioning Autism, Males and Females, Scientists and Mathematicians. Journal of Autism and Developmental Disorders, 31(1), 13.

Beckmann, C. F., DeLuca, M., Devlin, J. T., \& Smith, S. M. (2005). Investigations into resting-state connectivity using independent component analysis. Philos Trans R Soc Lond B Biol Sci, 360(1457), 1001-1013. doi:10.1098/rstb.2005.1634

Belardinelli, M. O., Palmiero, M., Sestieri, C., Nardo, D., Di Matteo, R., Londei, A., . . Romani, G. L. (2009). An $\mathrm{fMRI}$ investigation on image generation in different sensory modalities: the influence of vividness. Acta psychologica, 132(2), 190-200.

Bishop, D. V., \& Norbury, C. F. (2005). Executive functions in children with communication impairments, in relation to autistic symptomatology. 1: Generativity. Autism, 9(1), 7-27. doi:10.1177/1362361305049027

Bogousslavsky, J., Miklossy, J., Deruaz, J.-P., Assal, G., \& Regli, F. (1987). Lingual and fusiform gyri in visual processing: a clinico-pathologic study of superior altitudinal hemianopia. Journal of Neurology, Neurosurgery \& Psychiatry, 50(5), 607-614.

Bressler, S. L., \& Menon, V. (2010). Large-scale brain networks in cognition: emerging methods and principles. Trends Cogn Sci, 14(6), 277-290. doi:10.1016/j.tics.2010.04.004 
Calhoun, V. D., Adali, T., Pearlson, G., \& Pekar, J. J. (2001). Spatial and temporal independent component analysis of functional MRI data containing a pair of task-related waveforms. Hum Brain Mapp, 13(1), 4353 .

Carlson, S. M., \& Moses, L. J. (2001). Individual differences in inhibitory control and children's theory of mind. Child development, 72(4), 1032-1053.

Carlson, S. M., Moses, L. J., \& Breton, C. (2002). How specific is the relation between executive function and theory of mind? Contributions of inhibitory control and working memory. Infant and Child Development: An International Journal of Research and Practice, 11(2), 73-92.

Chmielewski, W. X., Yildiz, A., \& Beste, C. (2014). The neural architecture of age-related dual-task interferences. Front Aging Neurosci, 6, 193. doi:10.3389/fnagi.2014.00193

Chung, H. J., Weyandt, L. L., \& Swentosky, A. (2014). The physiology of executive functioning Handbook of executive functioning (pp. 13-27): Springer.

Constantino, J. N., Lajonchere, C., Lutz, M., Gray, T., Abbacchi, A., McKenna, K., . . Todd, R. D. (2006). Autistic social impairment in the siblings of children with pervasive developmental disorders. American Journal of Psychiatry, 163(2), 294-296.

Cordes, D., Haughton, V. M., Arfanakis, K., Wendt, G. J., Turski, P. A., Moritz, C. H., . . Meyerand, M. E. (2000). Mapping functionally related regions of brain with functional connectivity MR imaging. American Journal of Neuroradiology, 21(9), 1636-1644.

Corkin, S., Milner, B., \& Rasmussen, T. (1970). Somatosensory thresholds: Contrasting effects of postcentral-gyrus and posterior parietal-lobe excisions. Archives of Neurology, 23(1), 41-58.

Coxon, J. P., Stinear, C. M., \& Byblow, W. D. (2007). Selective inhibition of movement. Journal of neurophysiology, 97(3), 2480-2489.

Daly, E., Ecker, C., Hallahan, B., Deeley, Q., Craig, M., Murphy, C., .. Murphy, D. G. (2014). Response inhibition and serotonin in autism: a functional MRI study using acute tryptophan depletion. Brain, 137(Pt 9), 2600-2610. doi:10.1093/brain/awu178

de Wit, S. (2018). The Balance Between Goal-Directed and Habitual Action Control in Disorders of Compulsivity Goal-Directed Decision Making (pp. 331-365): Elsevier.

Di Martino, A., Ross, K., Uddin, L. Q., Sklar, A. B., Castellanos, F. X., \& Milham, M. P. (2009). Functional brain correlates of social and nonsocial processes in autism spectrum disorders: an activation likelihood estimation meta-analysis. Biol Psychiatry, 65(1), 63-74. doi:10.1016/j.biopsych.2008.09.022

Di Martino, A., Zuo, X. N., Kelly, C., Grzadzinski, R., Mennes, M., Schvarcz, A., . . Milham, M. P. (2013). Shared and distinct intrinsic functional network centrality in autism and attention-deficit/hyperactivity 
disorder. Biol Psychiatry, 74(8), 623-632. doi:10.1016/j.biopsych.2013.02.011

Duan, X., Chen, H., He, C., Long, Z., Guo, X., Zhou, Y., . . Chen, H. (2017). Resting-state functional underconnectivity within and between large-scale cortical networks across three low-frequency bands in adolescents with autism. Prog Neuropsychopharmacol Biol Psychiatry, 79(Pt B), 434-441. doi:10.1016/j.pnpbp.2017.07.027

Fan, L., Li, H., Zhuo, J., Zhang, Y., Wang, J., Chen, L., . . Jiang, T. (2016). The Human Brainnetome Atlas: A New Brain Atlas Based on Connectional Architecture. Cereb Cortex, 26(8), 3508-3526. doi:10.1093/cercor/bhw157

Foss-Feig, J. H., Adkinson, B. D., Ji, J. L., Yang, G., Srihari, V. H., McPartland, J. C., . . Anticevic, A. (2017). Searching for Cross-Diagnostic Convergence: Neural Mechanisms Governing Excitation and Inhibition Balance in Schizophrenia and Autism Spectrum Disorders. Biol Psychiatry, 81(10), 848-861. doi:10.1016/j.biopsych.2017.03.005

Geurts, H. M., van den Bergh, S. F., \& Ruzzano, L. (2014). Prepotent response inhibition and interference control in autism spectrum disorders: two meta-analyses. Autism Res, 7(4), 407-420.

doi:10.1002/aur.1369

Giuliano, A., Saviozzi, I., Brambilla, P., Muratori, F., Retico, A., \& Calderoni, S. (2018). The effect of age, sex and clinical features on the volume of Corpus Callosum in pre-schoolers with Autism Spectrum Disorder: a case-control study. Eur J Neurosci, 47(6), 568-578. doi:10.1111/ejn.13527

Hala, S., Hug, S., \& Henderson, A. (2003). Executive function and false-belief understanding in preschool children: Two tasks are harder than one. Journal of Cognition and Development, 4(3), 275-298.

Hashimoto, T., Tayama, M., Miyazaki, M., Murakawa, K., \& Kuroda, Y. (1993). Brainstem and cerebellar vermis involvement in autistic children. Journal of child neurology, 8(2), 149-153.

Haznedar, M. M., Buchsbaum, M. S., Hazlett, E. A., LiCalzi, E. M., Cartwright, C., \& Hollander, E. (2006). Volumetric analysis and three-dimensional glucose metabolic mapping of the striatum and thalamus in patients with autism spectrum disorders. Am J Psychiatry, 163(7), 1252-1263.

doi:10.1176/appi.ajp.163.7.1252

Herringshaw, A. J., Ammons, C. J., DeRamus, T. P., \& Kana, R. K. (2016). Hemispheric differences in language processing in autism spectrum disorders: A meta-analysis of neuroimaging studies. Autism Research, 9(10), 1046-1057.

Hoekstra, R. A., Bartels, M., Cath, D. C., \& Boomsma, D. I. (2008). Factor structure, reliability and criterion validity of the Autism-Spectrum Quotient (AQ): a study in Dutch population and patient groups. $J$ Autism Dev Disord, 38(8), 1555-1566. doi:10.1007/s10803-008-0538-x 
Humphrey, G., \& Dumontheil, I. (2016). Development of Risk-Taking, Perspective-Taking, and Inhibitory Control During Adolescence. Developmental Neuropsychology, 1-18.

Hurst, R. M., Mitchell, J. T., Kimbrel, N. A., Kwapil, T. K., \& Nelson-Gray, R. O. (2007). Examination of the reliability and factor structure of the Autism Spectrum Quotient (AQ) in a non-clinical sample. Personality and Individual Differences, 43(7), 1938-1949. doi:10.1016/j.paid.2007.06.012

Ingersoll, B., Hopwood, C. J., Wainer, A., \& Brent Donnellan, M. (2011). A comparison of three self-report measures of the broader autism phenotype in a non-clinical sample. J Autism Dev Disord, 41(12), 16461657. doi:10.1007/s10803-011-1192-2

Jahfari, S., Stinear, C. M., Claffey, M., Verbruggen, F., \& Aron, A. R. (2010). Responding with restraint: what are the neurocognitive mechanisms? J Cogn Neurosci, 22(7), 1479-1492. doi:10.1162/jocn.2009.21307

Just, M. A., Cherkassky, V. L., Keller, T. A., Kana, R. K., \& Minshew, N. J. (2006). Functional and Anatomical Cortical Underconnectivity in Autism: Evidence from an fMRI Study of an Executive Function Task and Corpus Callosum Morphometry. Cerebral Cortex, 17(4), 951-961.

Just, M. A., Cherkassky, V. L., Keller, T. A., Kana, R. K., \& Minshew, N. J. (2007). Functional and anatomical cortical underconnectivity in autism: evidence from an FMRI study of an executive function task and corpus callosum morphometry. Cereb Cortex, 17(4), 951-961. doi:10.1093/cercor/bhl006

Kana, R. K., Keller, T. A., Minshew, N. J., \& Just, M. A. (2007). Inhibitory control in high-functioning autism: decreased activation and underconnectivity in inhibition networks. Biol Psychiatry, 62(3), 198-206. doi:10.1016/j.biopsych.2006.08.004

Kana, R. K., Keller, T. A., Minshew, N. J., \& Just, M. A. (2007). Inhibitory Control in High-Functioning Autism: Decreased Activation and Underconnectivity in Inhibition Networks. Biological Psychiatry, 62(3), 198-206.

Kleinhans, N., Akshoomoff, N., \& Delis, D. C. (2005). Executive functions in autism and Asperger's disorder: flexibility, fluency, and inhibition. Dev Neuropsychol, 27(3), 379-401. doi:10.1207/s15326942dn2703_5

Kloosterman, P. H., Keefer, K. V., Kelley, E. A., Summerfeldt, L. J., \& Parker, J. D. (2011). Evaluation of the factor structure of the Autism-Spectrum Quotient. Personality and Individual Differences, 50(2), 310-314.

Lai, M. C., Lombardo, M. V., Chakrabarti, B., \& Baron-Cohen, S. (2013). Subgrouping the Autism "Spectrum": Reflections on DSM-5. PLoS biology, 11(4), e1001544.

Laidi, C., Boisgontier, J., Chakravarty, M. M., Hotier, S., d'Albis, M. A., Mangin, J. F., . . Houenou, J. (2017). Cerebellar anatomical alterations and attention to eyes in autism. Sci Rep, 7(1), 12008.

doi:10.1038/s41598-017-11883-w 
Langen, M., Leemans, A., Johnston, P., Ecker, C., Daly, E., Murphy, C. M., . . Consortium, A. (2012). Frontostriatal circuitry and inhibitory control in autism: Findings from diffusion tensor imaging tractography. Cortex, 48(2), 183-193. doi:10.1016/j.cortex.2011.05.018

Langen, M., Leemans, A., Johnston, P., Ecker, C., Daly, E., Murphy, C. M., .. Murphy, D. G. M. (2012). Fronto-striatal circuitry and inhibitory control in autism: Findings from diffusion tensor imaging tractography. Cortex, 48(2), 183-193. doi:10.1016/j.cortex.2011.05.018

Lau, W. Y.-P., Gau, S. S.-F., Chiu, Y.-N., Wu, Y.-Y., Chou, W.-J., Liu, S.-K., \& Chou, M.-C. (2013). Psychometric properties of the Chinese version of the Autism Spectrum Quotient (AQ). Research in developmental disabilities, 34(1), 294-305.

Lei, X., Wang, Y., Yuan, H., \& Mantini, D. (2014). Neuronal oscillations and functional interactions between resting state networks: effects of alcohol intoxication. Human brain mapping, 35(7), 3517-3528.

Lemon, J. M., Gargaro, B., Enticott, P. G., \& Rinehart, N. J. (2011). Executive functioning in autism spectrum disorders: a gender comparison of response inhibition. J Autism Dev Disord, 41(3), 352-356. doi:10.1007/s10803-010-1039-2

Logan, G. D., Cowan, W. B., \& Davis, K. A. (1984). On the ability to inhibit simple and choice reaction time responses: a model and a method. Journal of Experimental Psychology: Human Perception and Performance, 10(2), 276.

Luna, B., Doll, S. K., Hegedus, S. J., Minshew, N. J., \& Sweeney, J. A. (2007). Maturation of Executive Function in Autism. 61(4), 474-481.

McKinnon, C. J., Eggebrecht, A. T., Todorov, A., Wolff, J. J., Elison, J. T., Adams, C. M., . . Botteron, K. N. (2019). Restricted and repetitive behavior and brain functional connectivity in infants at risk for developing autism spectrum disorder. Biological Psychiatry: Cognitive Neuroscience and Neuroimaging, $4(1), 50-61$.

Mosconi, M. W., Kay, M., D'Cruz, A. M., Seidenfeld, A., Guter, S., Stanford, L. D., \& Sweeney, J. A. (2009). Impaired inhibitory control is associated with higher-order repetitive behaviors in autism spectrum disorders. Psychological Medicine, 39(9), 1559-1566. doi:10.1017/s0033291708004984

Oberle, E., Schonert-Reichl, K. A., Lawlor, M. S., \& Thomson, K. C. (2012). Mindfulness and Inhibitory Control in Early Adolescence. Journal of Early Adolescence, 32(4), 565-588.

Paakki, J. J., Rahko, J., Long, X., Moilanen, I., Tervonen, O., Nikkinen, J., . . Kiviniemi, V. (2010). Alterations in regional homogeneity of resting-state brain activity in autism spectrum disorders. Brain Res, 1321, 169179. doi:10.1016/j.brainres.2009.12.081

Padmanabhan, A., Garver, K., O'Hearn, K., Nawarawong, N., Liu, R., Minshew, N., . . Luna, B. (2015). Developmental Changes in Brain Function Underlying Inhibitory Control in Autism Spectrum Disorders. 
Pelphrey, K. A., Morris, J. P., \& McCarthy, G. (2004). Grasping the Intentions of Others: The Perceived Intentionality of an Action Influences Activity in the Superior Temporal Sulcus during Social Perception. Journal of Cognitive Neuroscience, 16(10), 1706-1716. doi:10.1162/0898929042947900

Power, J. D., Cohen, A. L., Nelson, S. M., Wig, G. S., Barnes, K. A., Church, J. A., . . Petersen, S. E. (2011). Functional network organization of the human brain. Neuron, 72(4), 665-678. doi:10.1016/j.neuron.2011.09.006

Rinehart, N. J., Bradshaw, J. L., Moss, S. A., Brereton, A. V., \& Tonge, B. J. (2008). Brief report: inhibition of return in young people with autism and Asperger's disorder. Autism, 12(3), 249-260. doi:10.1177/1362361307088754

Rutter, M. (2000). Genetic studies of autism: from the 1970s into the millennium. Journal of abnormal child psychology, 28(1), 3-14.

Sachs, O., Weis, S., Zellagui, N., Huber, W., Zvyagintsev, M., Mathiak, K., \& Kircher, T. (2008). Automatic processing of semantic relations in fMRI: Neural activation during semantic priming of taxonomic and thematic categories. Brain Research, 1218(none), 194-205.

Salmi, J., Roine, U., Glerean, E., Lahnakoski, J., Nieminen-von Wendt, T., Tani, P., . . Sams, M. (2013). The brains of high functioning autistic individuals do not synchronize with those of others. Neurolmage: Clinical, 3, 489-497. doi:10.1016/j.nicl.2013.10.011

Schmitt, L. M., Ankeny, L. D., Sweeney, J. A., \& Mosconi, M. W. (2016). Inhibitory Control Processes and the Strategies That Support Them during Hand and Eye Movements. Front Psychol, 7, 1927. doi:10.3389/fpsyg.2016.01927

Seeley, W. W., Menon, V., Schatzberg, A. F., Keller, J., Glover, G. H., Kenna, H., . . Greicius, M. D. (2007). Dissociable intrinsic connectivity networks for salience processing and executive control. J Neurosci, 27(9), 2349-2356. doi:10.1523/JNEUROSCI.5587-06.2007

Semrud-Clikeman, M., Walkowiak, J., Wilkinson, A., \& Butcher, B. (2010). Executive functioning in children with Asperger syndrome, ADHD-combined type, ADHD-predominately inattentive type, and controls. $J$ Autism Dev Disord, 4O(8), 1017-1027. doi:10.1007/s10803-010-0951-9

Sheikhani, A., Behnam, H., Mohammadi, M. R., Noroozian, M., \& Mohammadi, M. (2012). Detection of Abnormalities for Diagnosing of Children with Autism Disorders Using of Quantitative Electroencephalography Analysis. Journal of Medical Systems, 36(2), 957-963.

Shih, P., Keehn, B., Oram, J. K., Leyden, K. M., Keown, C. L., \& Muller, R. A. (2011). Functional differentiation of posterior superior temporal sulcus in autism: a functional connectivity magnetic resonance imaging study. Biol Psychiatry, 70(3), 270-277. doi:10.1016/j.biopsych.2011.03.040 
Smallwood, J., Brown, K., Baird, B., \& Schooler, J. W. (2012). Cooperation between the default mode network and the frontal-parietal network in the production of an internal train of thought. Brain Res, 1428, 60-70. doi:10.1016/j.brainres.2011.03.072

Stoeckel, C., Gough, P. M., Watkins, K. E., \& Devlin, J. T. (2009). Supramarginal gyrus involvement in visual word recognition. Cortex, 45(9), 1091-1096.

Takarae, Y., Luna, B., Minshew, N. J., \& Sweeney, J. A. (2014). Visual motion processing and visual sensorimotor control in autism. Journal of the international neuropsychological society, 20(1), 113-122.

Vaidya, C. J., Foss-Feig, J., Shook, D., Kaplan, L., Kenworthy, L., \& Gaillard, W. D. (2011). Controlling attention to gaze and arrows in childhood: an fMRI study of typical development and Autism Spectrum Disorders. Dev Sci, 14(4), 911-924. doi:10.1111/j.1467-7687.2011.01041.x

Vara, A. S., Pang, E. W., Doyle-Thomas, K. A., Vidal, J., Taylor, M. J., \& Anagnostou, E. (2014). Is inhibitory control a 'no-go' in adolescents with autism spectrum disorder? Molecular Autism, 5(1), 6.

Vink, M., Zandbelt, B. B., Gladwin, T., Hillegers, M., Hoogendam, J. M., van den Wildenberg, W. P., ... Kahn, R. S. (2014). Frontostriatal activity and connectivity increase during proactive inhibition across adolescence and early adulthood. Hum Brain Mapp, 35(9), 4415-4427. doi:10.1002/hbm.22483

Wang, Z., Wang, Y., Sweeney, J. A., Gong, Q., Lui, S., \& Mosconi, M. W. (2019). Resting-State Brain Network Dysfunctions Associated With Visuomotor Impairments in Autism Spectrum Disorder. Frontiers in Integrative Neuroscience, 13. doi:10.3389/fnint.2019.00017

Whitfield-Gabrieli, S., \& Nieto-Castanon, A. (2012). Conn: a functional connectivity toolbox for correlated and anticorrelated brain networks. Brain Connect, 2(3), 125-141. doi:10.1089/brain.2012.0073

Wilson, C. E., Happé, F., Wheelwright, S. J., Ecker, C., Lombardo, M. V., Johnston, P., . . Lai, M. C. The Neuropsychology of Male Adults With High-Functioning Autism or Asperger Syndrome. Autism Research, 7(5), 568-581.

Winston, C., Wilson, S. M., Mark, D. E., Kayser, A. S., Grossman, S. N., Pardis, P., . . Rankin, K. P. (2013). The salience network causally influences default mode network activity during moral reasoning. Brain(6), 6.

Xiao, T., Xiao, Z., Ke, X., Hong, S., Yang, H., Su, Y., . . Liu, Y. (2012). Response inhibition impairment in high functioning autism and attention deficit hyperactivity disorder: evidence from near-infrared spectroscopy data. Plos One, 7(10), e46569. doi:10.1371/journal.pone.0046569

Xiao, T., Xiao, Z., Ke, X., Hong, S., Yang, H., Su, Y., . . Liu, Y. (2012). Response Inhibition Impairment in High Functioning Autism and Attention Deficit Hyperactivity Disorder: Evidence from Near-Infrared Spectroscopy Data. Plos One, 7. 
Yan, C., \& Zang, Y. (2010). DPARSF: a MATLAB toolbox for "pipeline" data analysis of resting-state fMRI. Frontiers in Systems Neuroscience, 4(13). doi:10.3389/fnsys.2010.00013

\section{Tables}

Table1. A summary of the demographic information in the present study

\begin{tabular}{|llll|}
\hline & $\mathrm{N}=29$ & & \\
\hline Measure & Mean & $\mathrm{SD}$ & range \\
\hline Age & 19.21 & 0.41 & $19-20$ \\
\hline AQ(total) & 21.83 & 4.86 & $14-30$ \\
\hline Social skill & 4.14 & 2.10 & $1-8$ \\
\hline Attention switching & 5.55 & 2.08 & $1-9$ \\
\hline Attention to detail & 5.86 & 2.29 & $0-9$ \\
\hline Communication & 3.00 & 1.77 & $0-7$ \\
\hline Imagination & 3.34 & 1.14 & $1-6$ \\
\hline
\end{tabular}

Notes: $A Q=$ Autism Spectrum Quotient; $S D=$ standard deviation; $N=$ number

Table2. The partial correlation between AQ and SSRT value (one-tailed test)

\begin{tabular}{|c|c|c|c|c|c|c|c|}
\hline & $N=2 c$ & & & & & & \\
\hline Measure & 1 & 2 & 3 & 4 & 5 & 6 & 7 \\
\hline $1 \mathrm{SSRT}$ & - & & & & & & \\
\hline $2 \mathrm{AQ}$ (total) & $.044^{*}$ & - & & & & & \\
\hline 3 Social skill & .452 & .000 & - & & & & \\
\hline 4 Attention switching & $.003^{*}$ & .000 & .012 & - & & & \\
\hline 5Attention to detail & .404 & .139 & .040 & .371 & - & & \\
\hline 6 Communication & .051 & .000 & .001 & .026 & .091 & - & \\
\hline 7 Imagination & .483 & .135 & .344 & .451 & .141 & .15 & \\
\hline
\end{tabular}

Notes: $A Q=$ Autism Spectrum Quotient $;{ }^{*} p<0.05 . S S R T=$ Stop Signal Reaction Time; $N=$ number

Table3. Seed network to whole-brain connectivity analysis at stop success $>$ base condition 


\begin{tabular}{|c|c|c|c|}
\hline Seed network & Cluster size & MNI coordinates & $\mathrm{P}_{-\mathrm{FWE}}$ \\
\hline \multicolumn{4}{|l|}{ LN } \\
\hline Vermis $45(+)$ & 202 & $0,-52,6$ & 0.03 \\
\hline ITG_L (-) & 172 & $-54,-46,-16$ & 0.01 \\
\hline \multicolumn{4}{|l|}{ SN } \\
\hline SMG_L (-) & 425 & $-54,-42,44$ & 0.006 \\
\hline \multicolumn{4}{|l|}{ DAN } \\
\hline LG_L (-) & 466 & $-12,-72,2$ & 0.004 \\
\hline \multicolumn{4}{|l|}{ FPN } \\
\hline PCG_L (+) & 178 & $-20,-40,56$ & 0.008 \\
\hline
\end{tabular}

Notes: salience network (LN)); salience network (SN); dorsal attention network (DAN); frontoparietal network (FPN); inferior temporal gyrus (ITG); supramarginal gyrus (SMG); lingual gyrus (LG); postcentral gyrus (PCG); L: left; R: right; MNI: Montreal Neurological Institute.

Table 4. ROI-to-ROI functional connectivity analysis at stop success $>$ base condition 


\begin{tabular}{|c|c|c|c|c|c|}
\hline ROI & $\begin{array}{l}\text { Approximate brain } \\
\text { area }\end{array}$ & $\begin{array}{l}\text { Power atlas } \\
\text { network } \\
\text { assignment }\end{array}$ & MNI coordinates & Statistic & $\mathrm{P}_{\mathrm{FDR}}$ \\
\hline $\begin{array}{l}(69)^{a_{-}} \\
(209)\end{array}$ & SMG_L-insula_R & $S N-S N$ & $\begin{array}{l}(-53,-22,23)(36,22 \\
3)\end{array}$ & 6.12 & 0.0005 \\
\hline $\begin{array}{l}(134)^{-} \\
(255)^{\mathrm{a}}\end{array}$ & $\begin{array}{l}\text { Precuneus_L- } \\
\text { PCG_R }\end{array}$ & Memory - FPN & $\begin{array}{l}(-7,-71,42)(47 \\
-30,49)\end{array}$ & -5.35 & 0.0040 \\
\hline $\begin{array}{l}(80)- \\
(124)\end{array}$ & MOG_R - PHG_L & DMN - DMN & $\begin{array}{l}(43,-72,28)(-26,-40 \\
-8)\end{array}$ & 5.00 & 0.0101 \\
\hline $\begin{array}{l}(191)^{-} \\
(117)^{\mathrm{a}}\end{array}$ & IPL_L -MTG_L & DAN-DMN & $\begin{array}{l}(-28,-58,48)(-56,-13 \\
-10)\end{array}$ & 4.85 & 0.0151 \\
\hline $\begin{array}{l}(263)^{-} \\
(79)^{\mathrm{a}}\end{array}$ & SPL_L - MTG_L & DAN - DMN & $\begin{array}{l}(-17,-59,64)(-46,-61 \\
21)\end{array}$ & 4.78 & 0.0180 \\
\hline $\begin{array}{l}(89)^{-} \\
(255)^{\mathrm{a}}\end{array}$ & $\begin{array}{l}\text { Precuneus_R - } \\
\text { PCG_R }\end{array}$ & DMN - FPN & $\begin{array}{l}(6,-59,35)(47,-30 \\
49)\end{array}$ & -4.78 & 0.0182 \\
\hline $\begin{array}{l}(110)- \\
(226)\end{array}$ & $\begin{array}{l}\text { OFC_R - } \\
\text { Brainstem_L }\end{array}$ & $\begin{array}{l}\text { DMN- } \\
\text { Subcortical }\end{array}$ & $(8,42,-5)(-5,-28,-4)$ & -4.45 & 0.0427 \\
\hline $\begin{array}{l}(239)^{-} \\
(151)^{\mathrm{a}}\end{array}$ & MTG_R - LG_L & VAN - Visual & $\begin{array}{l}(51,-29,-4)(-15,-71 \\
-8)\end{array}$ & 4.39 & 0.0499 \\
\hline
\end{tabular}

Notes: ROI are defined by Power Atlas; ${ }^{a}$ identified in seed network to whole-brain analysis

SMG: supramarginal gyrus; PCG: postcentral gyrus; MOG: middle occipital gyrus; PHG: parahippocampal gyrus; IPL: inferior parietal gyrus; MTG: middle temporal gyrus; SPL: superior parietal gyrus; OFC: orbitalfrontal cortex; $L G$ : lingual gyrus SN: salience network; FPN: frontoparietal network; DMN: default mode network; DAN: dorsal attention network; VAN: ventral attention network. L: left; R: right; MNI: Montreal Neurological Institute.

Table5 The correlation between $\mathrm{AQ}$ total and sub-dimensions and brain areas 


\begin{tabular}{|lllll|}
\hline Items & PCG & ITG & SMG & LG \\
\hline AQ total & $0.000 / 0.694$ & $0.000 /-0.686$ & $0.000 /-0.669$ & $0.003 /-0.529$ \\
\hline Social skill & $0.002 / 0.547$ & - & - & - \\
\hline Attention switching & $0.000 / 0.654$ & $0.008 /-0.481$ & $0.005 /-0.503$ & - \\
\hline Attention to detail & - & $0.034 /-0.395$ & - & - \\
\hline communication & $0.024 / 0.415$ & $0.028 /-0.408$ & $0.003 /-0.536$ & $0.033 /-0.397$ \\
\hline imagination & - & - & - & - \\
\hline
\end{tabular}

Note: $p / r$.

\section{Figures}

\section{Go trail}

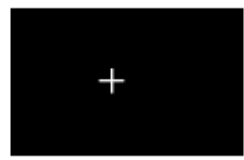

$300 \mathrm{~ms}$

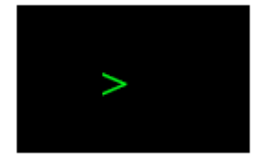

$500 \mathrm{~ms}$

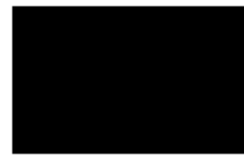

$700 \mathrm{~ms}$

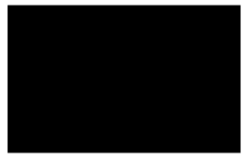

1-4s (Jitter)

Stop trail

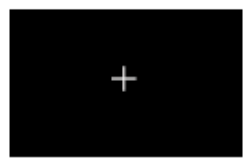

$300 \mathrm{~ms}$

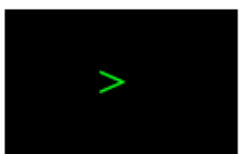

SSD ms

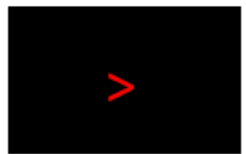

$300 \mathrm{~ms}$

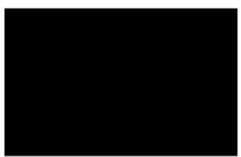

900-SSD ms

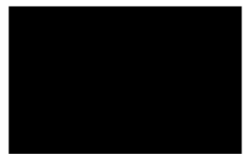

1-4s(Jitter)

\section{Figure 1}

The stop-signal task. Each trail began with a central fixation cross during $300 \mathrm{~ms}$, then a green arrow pointing left or right was displayed on the screen. For the Go trail, when appeared green left arrow lasted for $500 \mathrm{~ms}$, participants were asked to respond by pressing the " 1 ", or " 2 " button when appeared green right arrow within $1500 \mathrm{~ms}$, then the blank screen lasted for $700 \mathrm{~ms}$. For the Stop trail, participants were asked to withhold the response they already initiated. The jitter between trails ranged from $1 \mathrm{~s}$ to $4 \mathrm{~s}$ (mean, 2.5s). Which consists of 32 Stop trials and $96 \mathrm{Go}$ trials. The duration of the task was 8 min $46 \mathrm{~s}$. 


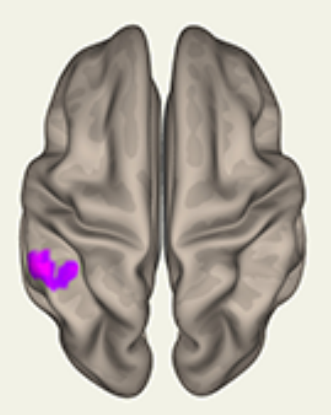

SH - SMG_L

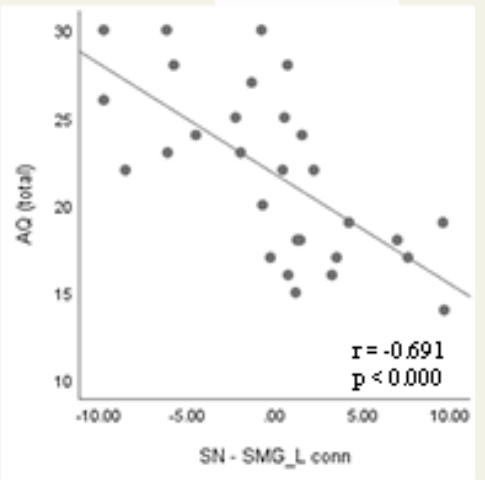

SN - SMG_L conn

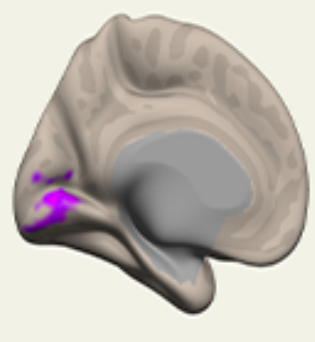

DAF - LG_R

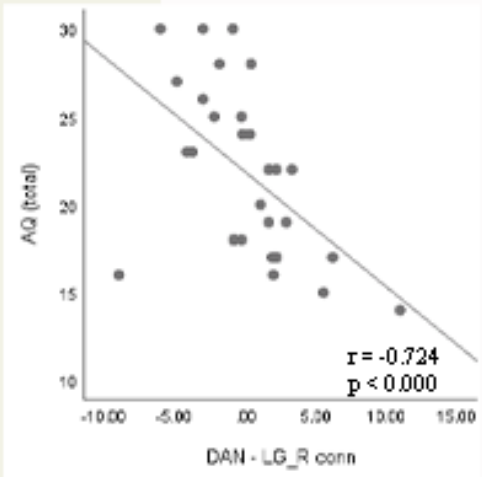

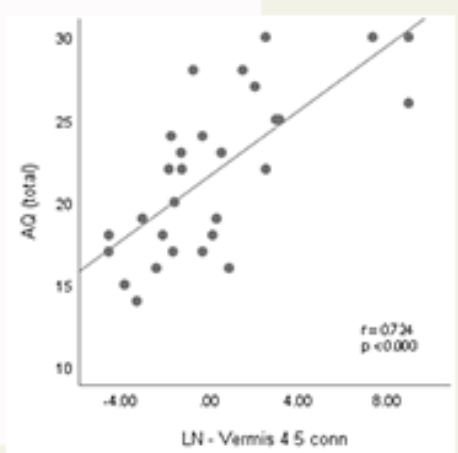

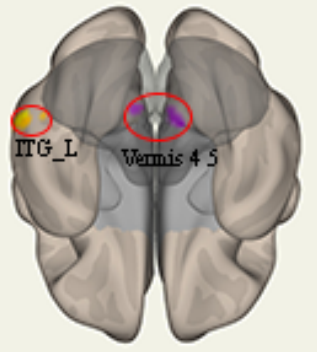

LN - Vermis 4 SITG -L

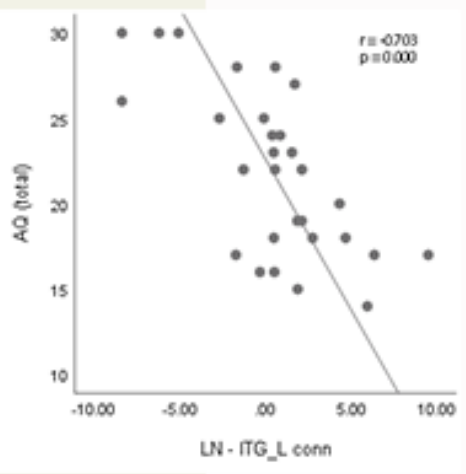

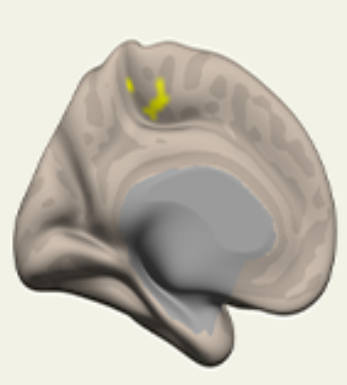

FPH - PCG_L

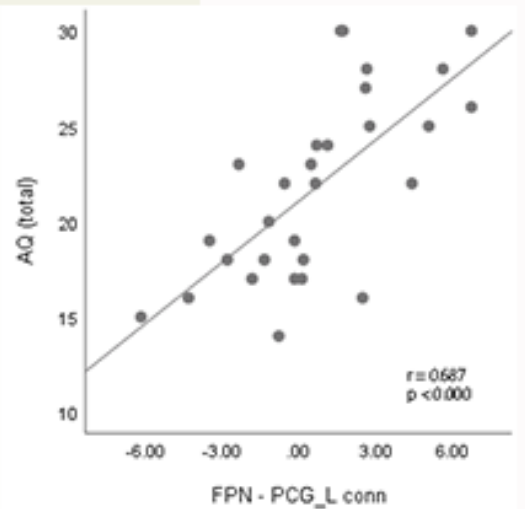

\section{Figure 2}

The take network as seed to voxel analysis showed that the saliance network (SN) has a significant nagetive connection with left supramarginal gyrus (SMG); dorsal attention network (DAN) has significant negative connection with left lingural gyrus (LG); frontoparietal network (FPN) has a significant positive connection with left postcentral gyrus (PCG); lauguage network (LN) have significant positive connection with vermis 45 and negatice connection with left inferior temporal gyrus (ITG). 

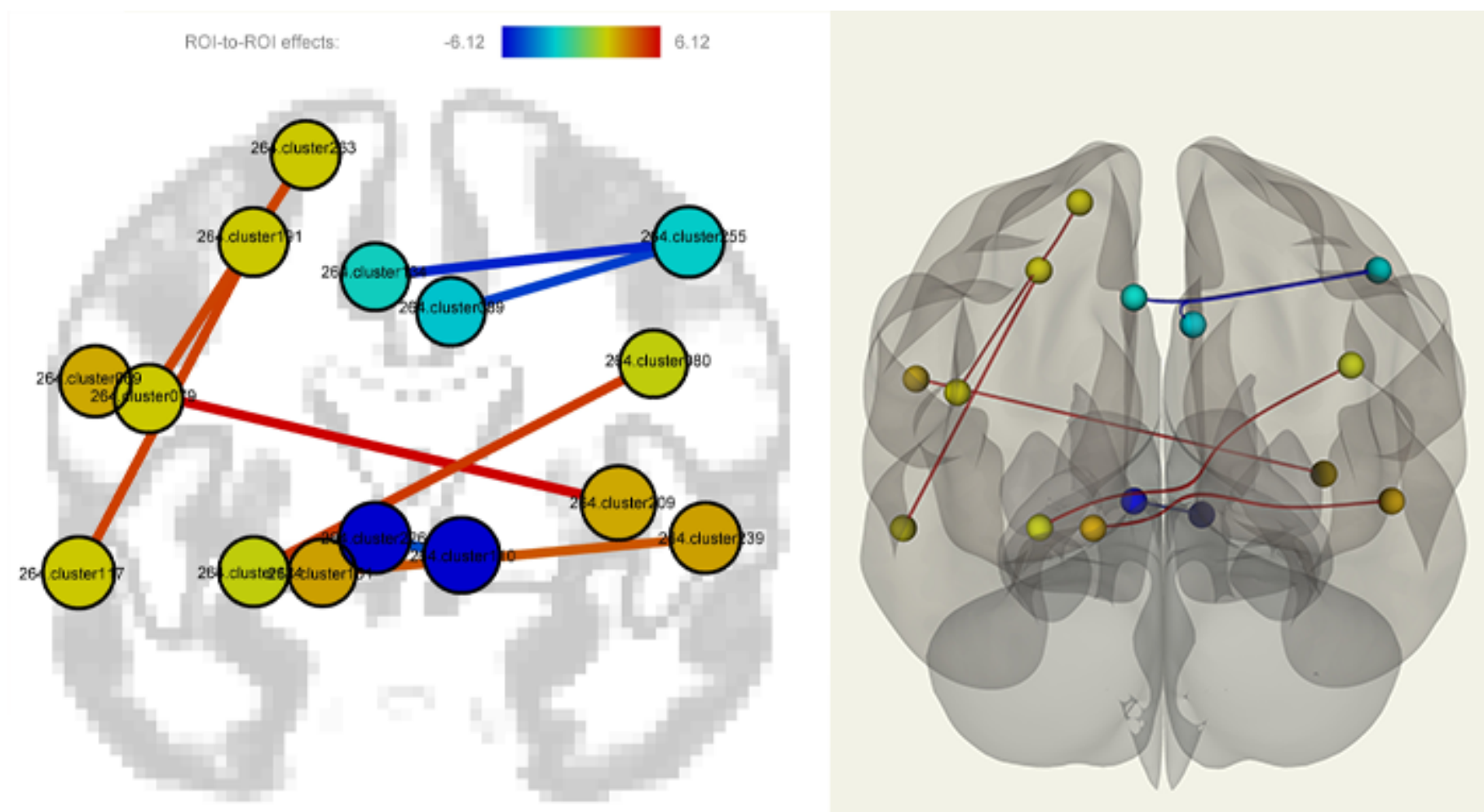

Figure 3

In ROI-to-ROI level, we found three negative connections and four positive connections, supramarginal gyrus (SMG), postcenral gyrus (PCG), lingural gyrus (LG), and temporal gyrus are also included in seed network to whole-brain analysis. The red curve shows positive functional connectivity, The blue curve shows negative functional connectivity. 